\title{
Tumour suppression by a protein hydroxylase
}

A. Zayer ${ }^{1}$, H. Smith, M. Pillai ${ }^{3}$, R. Sekirnik ${ }^{4}$, J. Zak ${ }^{5}$, X. Lu ${ }^{5}$, C. Schofield ${ }^{4}$, M. Bix ${ }^{6}$, P. Ratcliffe ${ }^{7}$, M. Coleman².

${ }^{1}$ University of Cambridge, University of Cambridge, Cambridge, United Kingdom, ${ }^{2}$ University of Birmingham, Institute of Cancer and Genomic Sciences, Birmingham, United Kingdom, ${ }^{3}$ St Jude Childrens Hospital, Department of Immunology, Memphis, USA, ${ }^{4}$ University of Oxford, Chemistry Research Laboratories, Oxford, United Kingdom, ${ }^{5}$ University of Oxford, Ludwig Institute for Cancer Research, Oxford, United Kingdom, ${ }^{6}$ Chiba University, Dept. of Immunology, Chiba, Japan, ${ }^{7}$ University of Oxford, Nuffield Department of Medicine, Oxford, United Kingdom

Introduction: Hydroxylation is an emerging modification catalysed by a family of '2-oxoglutarate (2OG)-dependent oxygenases' that includes the Collagen and HIF hydroxylases, TET 5hmC demethylases, and JmjC histone demethylases. These enzymes are widely implicated in cancer due to roles in hypoxia signalling, extracellular matrix formation, and epigenetics, and because of their inhibition by oncometabolites. Although other sub-families of 20G-oxygenases exist they are less well characterised and their role in cancer remains largely unexplored. We are defining the biochemical targets and biological functions of $\mathrm{JmjC}$ protein hydroxylases, a poorly characterised family of 2OG-oxygenases related to the JmjC histone demethylases, and have discovered that these enzymes regulate fundamental biological processes that are commonly deregulated in cancer. MycInduced Nuclear Antigen (MINA) is a JmjC protein hydroxylase that we have shown promotes ribosomal 60S subunit abundance through modification of Rpl27a. Consistent with a role in supporting growth and ribosome biogenesis, MINA is overexpressed in several tumour types. However, our recent work suggests MINA also has the capacity for tumour suppressor activity. Here we describe our investigation of the MINA protein hydroxylase and its tumour suppressor role.

Results: Anchorage-independent growth assays indicate that reconstitution of MINA into knockout K-Ras-transformed cells and MINA mutant human tumour epithelial cell line suppresses transformation. These suppressor affects are recapitulated in xenograft tumour growth assays and in cancer models using transgenic MINA knockout mice. Consistent with the possibility of MINA inactivation in human tumours, cancer sequencing databases include nonsense, frameshift, splice site and missense mutations across a variety of tumour types. Importantly, missense mutations are clustered in 'hotspots' within functionally important domains and can inhibit hydroxylase activity.

Conclusion: Our work demonstrates that MINA is a novel class of tumour suppressor, and indicates that further investigation into the roles of JmjC protein hydroxylases in cancer is warranted. 